\title{
MDM2 is a novel E3 ligase for HIV-I Vif
}

Taisuke Izumi ${ }^{1}$, Akifumi Takaori-Kondo*1, Kotaro Shirakawa ${ }^{1,2}$, Hiroaki Higashitsuji ${ }^{3}$, Katsuhiko Itoh ${ }^{3}$, Katsuhiro Io $^{1}$, Masashi Matsui ${ }^{1}$, Kazuhiro Iwai ${ }^{4}, 5$, Hiroshi Kondoh ${ }^{6}$, Toshihiro Sato ${ }^{7}$, Mitsunori Tomonaga ${ }^{7}$, Satoru Ikeda ${ }^{7}$, Hirofumi Akari ${ }^{8}$, Yoshio Koyanagi ${ }^{9}$, Jun Fujita ${ }^{3}$ and Takashi Uchiyama ${ }^{1}$

\author{
Address: ${ }^{1}$ Department of Hematology and Oncology, Graduate School of Medicine, Kyoto University, 54 Shogoin-Kawaracho, Sakyo-ku, Kyoto

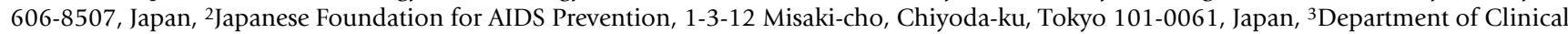 \\ Molecular Biology, Graduate School of Medicine, Kyoto University, 54 Shogoin-Kawaracho, Sakyo-ku, Kyoto 606-8507, Japan, ${ }^{4}$ Department of \\ Molecular Cell Biology, Graduate School of Medicine, Osaka City University, 1-4-3 Asahi-machi, Abeno-ku, Osaka 545-8585, Japan, ${ }^{5}$ CREST, \\ Japan Science Technology Corporation, Kawaguchi, Saitama 332-0012, Japan, ${ }^{6}$ Department of Geriatric Medicine, Graduate School of Medicine, \\ Kyoto University, 54 Shogoin-Kawaracho, Sakyo-ku, Kyoto 606-8507, Japan, ${ }^{7}$ Central Pharmaceutical Research Institute, Japan Tobacco Inc., $1-1$ \\ Murasaki-cho, Takatsuki, Osaka 569-1125, Japan, ${ }^{8}$ Laboratory of Disease Control, Tukuba Primate Research Center, National Institute of \\ Biomedical Innovation, Hachimandai-1, Tsukuba, Ibaraki 305-0843, Japan and 'Laboratory of Viral Pathgenesis, Institute for Virus Research, \\ Kyoto University, 53 Shogoin-Kawaracho, Sakyo-ku, Kyoto 606-8507, Japan \\ Email: Taisuke Izumi - izumi.t@aw3.ecs.kyoto-u.ac.jp; Akifumi Takaori-Kondo* - atakaori@kuhp.kyoto-u.ac.jp; \\ Kotaro Shirakawa - kotash@kuhp.kyoto-u.ac.jp; Hiroaki Higashitsuji - hhigashi@virus.kyoto-u.ac.jp; Katsuhiko Itoh - katsu@virus.kyoto- \\ u.ac.jp; Katsuhiro Io - katsu829@kuhp.kyoto-u.ac.jp; Masashi Matsui - mmatsui@kuhp.kyoto-u.ac.jp; \\ Kazuhiro Iwai - kiwai@cellbio.med.osaka-u.ac.jp; Hiroshi Kondoh - hkondoh@kuhp.kyoto-u.ac.jp; \\ Toshihiro Sato - toshihiro.sato@ims.jti.co.jp; Mitsunori Tomonaga - mitsunori.tomonaga@ims.jti.co.jp; \\ Satoru Ikeda - satoru.ikeda@ims.jti.co.jp; Hirofumi Akari - akari@nibio.go.jp; Yoshio Koyanagi - ykoyanag@virus.kyoto-u.ac.jp; \\ Jun Fujita - jfujita@virus.kyoto-u.ac.jp; Takashi Uchiyama - uchiyata@kuhp.kyoto-u.ac.jp \\ * Corresponding author
}

Published: 7 January 2009

Retrovirology 2009, 6:1 doi:10.1186/1742-4690-6-1
Received: 16 September 2008

Accepted: 7 January 2009

This article is available from: http://www.retrovirology.com/content/6/I/I

(C) 2009 Izumi et al; licensee BioMed Central Ltd.

This is an Open Access article distributed under the terms of the Creative Commons Attribution License (http://creativecommons.org/licenses/by/2.0), which permits unrestricted use, distribution, and reproduction in any medium, provided the original work is properly cited.

\begin{abstract}
The human immunodeficiency virus type I (HIV-I) Vif plays a crucial role in the viral life cycle by antagonizing a host restriction factor APOBEC3G (A3G). Vif interacts with A3G and induces its polyubiquitination and subsequent degradation via the formation of active ubiquitin ligase (E3) complex with Cullin5-ElonginB/C. Although Vif itself is also ubiquitinated and degraded rapidly in infected cells, precise roles and mechanisms of Vif ubiquitination are largely unknown. Here we report that MDM2, known as an E3 ligase for p53, is a novel E3 ligase for Vif and induces polyubiquitination and degradation of Vif. We also show the mechanisms by which MDM2 only targets Vif, but not A3G that binds to Vif. MDM2 reduces cellular Vif levels and reversely increases A3G levels, because the interaction between MDM2 and Vif precludes A3G from binding to Vif. Furthermore, we demonstrate that MDM2 negatively regulates HIV-I replication in non-permissive target cells through Vif degradation. These data suggest that MDM2 is a regulator of HIV-I replication and might be a novel therapeutic target for anti-HIV-I drug.
\end{abstract}




\section{Background}

Host restriction factors protect hosts from viruses, whereas viruses evade these proteins to replicate more efficiently in host cells. The interplay between the host restriction factors and viral proteins is therefore very important for regulating viral replication $[1,2]$. A3G (Apolipoprotein B mRNA editing enzyme, catalytic polypeptide-like $3 \mathrm{G}$ ) is a newly identified anti-HIV-1 host factor [3], which belongs to the APOBEC superfamily of cytidine deaminases, consisting of APOBEC1, APOBEC2, AID (activation-induced cytidine deaminase), APOBEC3(A-H), and APOBEC4 [4]. A3G is incorporated into HIV-1 virions and inhibits HIV-1 replication by inducing G-to-A hypermutation in viral cDNA during reverse transcription [5-8]. HIV-1 Vif counteracts A3G by targeting it for proteasomal degradation, thus supporting HIV-1 replication in non-permissive target cells [9-11]. Vif forms a ubiquitin ligase (E3) complex with Cullin5 (Cul5), Elongin B, and Elongin C and functions as a substrate recognition subunit of this complex to induce ubiquitination and subsequent degradation of A3G $[12,13]$. Vif also counteracts several APOBEC3 proteins including APOBEC3F (A3F) [14,15]. These observations reconcile the long-standing mystery of why Vif function is necessary for HIV-1 to infect non-permissive cells. On the other hand, it has been shown that intracellular levels of Vif are maintained relatively low by ubiquitination in virus-producing cells [16-18]. Although several groups have reported E3 ligases important for Vif ubiquitination $[17,18]$, the precise roles and mechanisms of Vif ubiquitination remain unclear. Here we demonstrate that MDM2 is a novel E3 ligase for Vif and that it induces ubiquitination and degradation of Vif, thereby regulating HIV-1 replication.

\section{Results \\ MDM2 downregulates cellular Vif levels by inducing its degradation in a proteasome-dependent manner}

To investigate the biological roles and molecular mechanisms of Vif ubiquitination, we tried to identify a novel E3 ligase that may be involved in the ubiquitination of Vif. During a search for Vif-interacting proteins in the HIV, Human Protein Interaction Database of National Institute for Allergy \& Infectious Diseases http:// www.ncbi.nlm.nih.gov/RefSeq/HIVInteractions/, we were struck by a protein called Gankyrin (proteasome 26S subunit, non-ATPase, 10 (PSMD10)). We first examined the biological effects of Gankyrin, but could not detect a downregulation of Vif (data not shown). As we previously reported that Gankyrin itself doesn't have an enzymatic activity and that it rather enhances the E3 ligase activity of MDM2 on p53 ubiquitination and degradation as a cofactor [19], we tested the possibility that MDM2 plays an important role in Vif ubiquitination as a novel E3 ligase. We examined the effect of several E3 ligases including
MDM2 (a RING finger type E3 that mediates p53 ubiquitination and degradation [20]), Cul5 (another RING finger type E3 that forms a complex with Vif and is reported to induce Vif ubiquitination $[17,21])$, and Parkin (another RING finger type E3) on cellular Vif levels (Fig. 1A). HEK293T cells were transfected with a subgenomic expression vector pNL-A1 that expressed all HIV-1 proteins except for gag and pol products [22], together with the expression plasmids for these E3 ligases. We found that the ectopic expression of MDM2 downregulated the cellular levels of Vif as well as p53 in transfected cells in a dose-dependent manner (Fig. 1A, lanes 8-10), whereas Parkin and Cul5 did not affect their cellular levels (lanes 2-4 and 5-7, respectively), even though the latter proteins were expressed more than MDM2. Our results are discrepant with previous reports that demonstrated $\mathrm{Cul} 5$ induced Vif ubiquitination and degradation $[17,23]$. We assume that overexpression of Cul5 alone is insufficient to induce Vif degradation, because other E3 components are not overexpressed. Ectopic expression of MDM2 did not affect cellular levels of another viral protein such as Nef, suggesting that MDM2 specifically downregulated Vif levels; this result also excluded the possibility that MDM2 affected the transcriptional activity of the HIV-1 LTR.

Because it is well known that MDM2 regulates p53 levels by modulating its protein stability, we next examined the protein stability of Vif with the ectopic expression of MDM2. HEK293T cells were transfected with pNL-A1 with or without a MDM2 expression vector and treated with cycloheximide $21 \mathrm{hrs}$ after transfection. After cycloheximide treatment, cellular levels of Vif decreased by $60 \%$ in MDM2-transfected cells and by $20 \%$ in control cells, respectively (Fig. 1B \&1C), indicating that Vif decayed much faster when MDM2 was overexpressed. The stability profile of Vif protein was similar to that of p53 (Fig. 1B). However, in our hands, the half-life of Vif protein was longer than those shown in previous studies from several laboratories. We interpret that this difference is attributable to divergent methods used in the studies which employed radioisotopes or cycloheximide. Thus, our findings suggest that MDM2 affects the stability of Vif protein similar to its effect on p53. We also examined the stability of Vif in MDM2-/- MEF cells. Vif decayed much faster in p53-/- MEF cells than in p53-/-MDM2-/- double knock-out (DKO) MEF cells (Additional file 1), suggesting that endogenous MDM2 can also influence the stability of Vif. We then tested a RING finger domain-deleted MDM2 mutant, $\triangle \mathrm{RF}$, which is inactive for the ubiquitination activity of MDM2 [24]. Ectopic expression of MDM2 suppressed cellular Vif levels, but the expression of $\Delta \mathrm{RF}$ did not (Fig. 1D). This result suggests that ubiquitination of Vif by MDM2 is involved in the downregulation of cellular Vif levels. We further treated transfected cells with a proteasome inhibitor MG132 to see whether the down- 


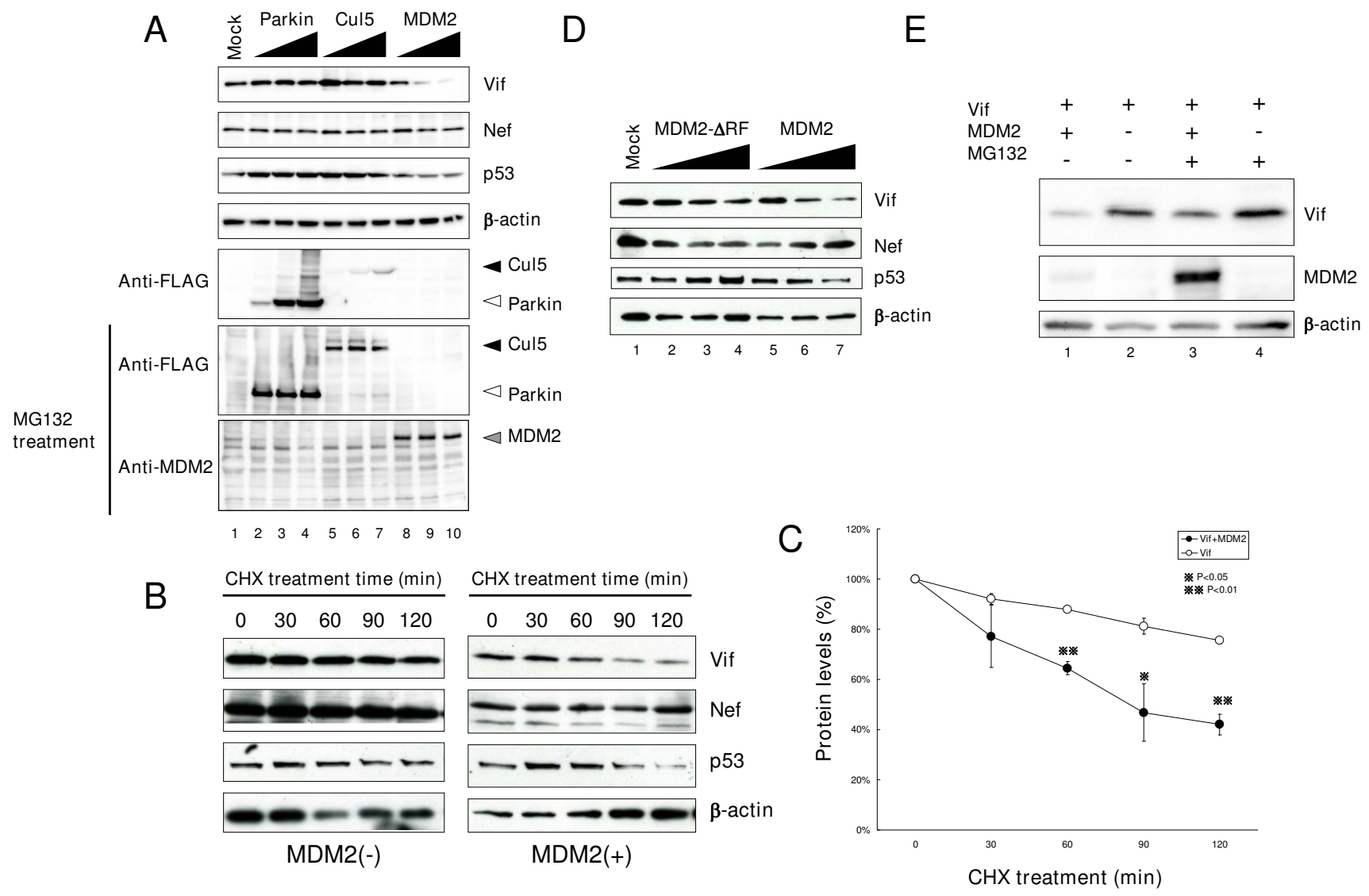

\section{Figure I}

MDM2 downregulated cellular Vif levels in a proteasome dependent manner. (A) MDM2 reduced cellular levels of Vif as well as p53, but not that of Nef. HEK293T cells were cotransfected with expression vectors for the indicated E3 ligases and a subgenomic HIV-I expression vector pNL-AI. Cell lysates were subjected to immunoblotting with the indicated Abs. We could not detect the expression of FLAG-MDM2 without MGI32 treatment, because of a rapid degradation of MDM2. MGI32 treatment enabled us to detect expression of MDM2 only with anti-MDM2 Ab, but not with anti-FLAG mAb. (B) Twenty-two hours after transfection, the cells were treated with cycloheximide $(\mathrm{CHX})(80 \mu \mathrm{g} / \mathrm{ml})$ for the indicated times, and cell lysates were subjected to immunoblotting with the indicated Abs. (C) The amounts of Vif and Nef were quantified by densitometry, and Vif protein levels were calculated using Nef protein levels as normalizing loading controls and presented as percentage values relative to that without $\mathrm{CHX}$ treatment set as $100 \%$. Values are presented as averages of three independent experiments. (D) MDM2 downregulated Vif, but a $\triangle R F$ mutant did not. HEK293T cells were cotransfected with expression vectors for MDM2 and the mutant together with pNL-AI, and cell lysates were subjected to immunoblotting with the indicated Abs. (E) p53-/-MDM2-/- DKO-MEF cells were cotransfected with expression vectors for MDM2 and Vif, and treated with $10 \mu \mathrm{M}$ MGI 32 for $6 \mathrm{hrs}$, and cell lysates were subjected to immunoblotting with the indicated Abs.

regulation of Vif by MDM2 was proteasome-dependent. Treatment with MG132 clearly restored the cellular Vif level that was downregulated by MDM2 (Fig. 1E, top panel, lane 3 as compared with lane 1), supporting that the MDM2-mediated downregulation of Vif was proteasome-dependent. Taken together, we concluded that MDM2 downregulates cellular Vif level by inducing its degradation in a proteasome-dependent manner.

\section{MDM2 specifically binds and downregulates Vif}

To further investigate the molecular link between MDM2 and Vif, we next examined the physical interaction of MDM2 with Vif. Immunoprecipitation assays showed that Vif was co-precipitated with MDM2 (Fig. 2A). Glutathione S-transferase (GST) pull-down assays showed that MDM2 was found in GST-Vif-bound, but not GSTbound, material (data not shown). Using a series of MDM2 deletion mutants, we determined that the central region of MDM2 (amino acids 168-320) was necessary for Vif binding (Fig. 2B, left panel \&2C). To more precisely 


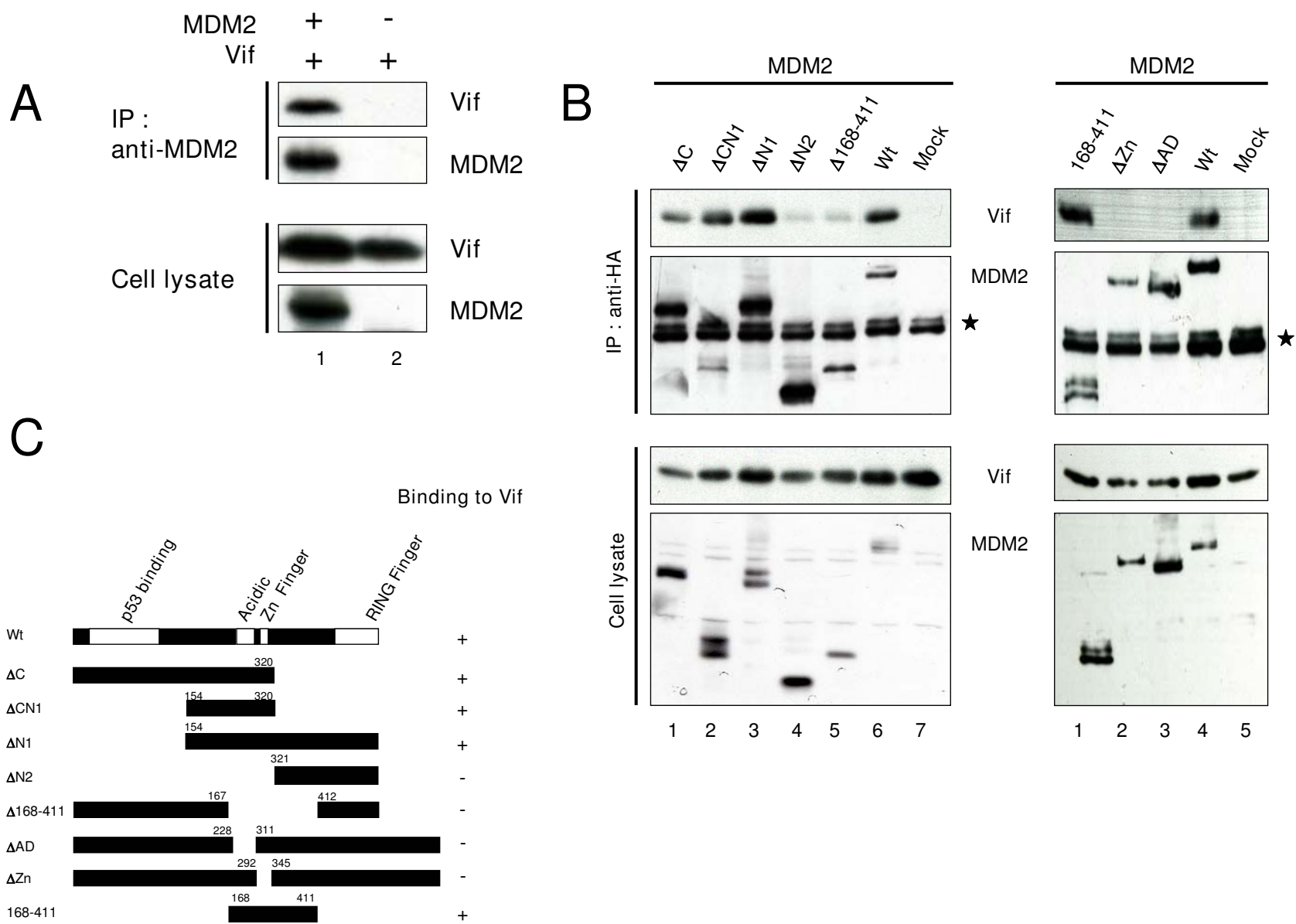

Figure 2

MDM2 bound Vif in its central domain. (A) Immunoprecipitation assays revealed the interaction of MDM2 with Vif in vivo. HEK293T cells were cotransfected with expression vectors for MDM2 and Vif and treated with MGI 32 for 6 hrs prior to harvest. Cell lysates were immunoprecipitated with anti-MDM2 mAb followed by immunoblotting with the indicated Abs (upper two panels). Cell lysates were also subjected to immunoblotting with the indicated Abs (lower two panels). (B) The interaction domain of MDM2 with Vif. HEK293T cells were cotransfected with expression vectors for HA-tagged MDM2 wild type (Wt) and mutants together with pNL-AI, and cell lysates were immunoprecipitated with anti-HA mAb followed by immunoblotting with the indicated Abs. Asterisk indicates immunoglobulin heavy chains from thenimmunoprecipitation. (C) Schematics of MDM2 mutants binding to Vif are shown.

determine a Vif-binding domain, we further tested mutants deleted in a $\mathrm{Zn}$ Finger domain $(\Delta \mathrm{Zn})$ or in an acidic domain $(\triangle \mathrm{AD})$. Neither mutant could bind Vif, whereas the mutant containing amino acids $168-411$ was able to bind Vif, suggesting that both domains are necessary and that the central domain is sufficient for Vif binding (Fig. 2B, right panel \&2C). Additionally, using a series of Vif deletion mutants, we also found that the N-terminal region of Vif (amino acids 4-22) is needed for MDM2 binding (Fig. 3A \&3C). Furthermore, we examined the MDM2-mediated downregulation of Vif mutants. MDM2 was able to efficiently downregulate cellular levels of the
MDM2-binding Vif mutants but not that of an MDM2non binding mutant, $\Delta 4-45$ (Fig. $3 \mathrm{~B}$ ). Collectively, these results indicated that the Vif-MDM2 interaction is required for MDM2-mediated downregulation of Vif (Fig. 3C).

\section{MDM2 induces ubiqutination of Vif}

Since we found that MDM2 bound Vif and promoted its degradation via a proteasomal pathway, we next examined whether MDM2 is involved in the polyubiquitination of Vif. In vitro ubiquitination assays revealed that bacterially expressed GST-MDM2 was able to induce the 


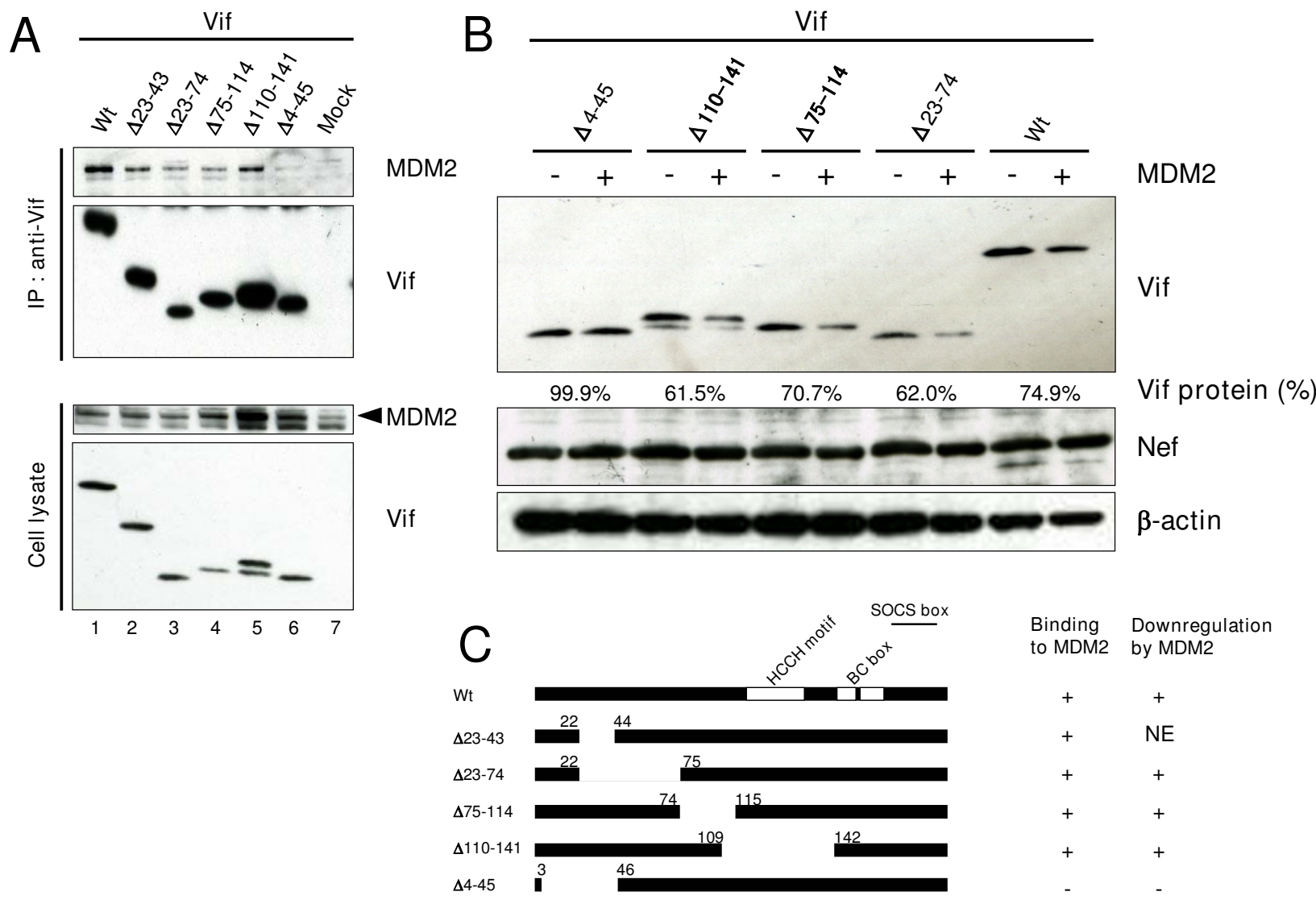

Figure 3

MDM2 specifically bound and downregulated Vif. (A) The interaction domain of Vif with MDM2. HEK293T cells were cotransfected with expression vectors for Vif and mutants together with PCMV/HA-MDM2, and cell lysates were immunoprecipitated with anti-Vif $m A b$ followed by immunoblotting with the indicated Abs. Arrowhead indicates MDM2. (B) The downregulation of Vif protein by MDM2. HEK293T cells were cotransfected with expression vectors for Vif and mutants with or without PCMV/HA-MDM2, and cell lysates were subjected to immunoblotting with the indicated Abs. The amounts of Vif were quantified by densitometry and shown as the protein ratio relative to that without expression of MDM2. (C) Schematics of Vif mutants bound by and downregulated by MDM2. NE: not examined.

polyubiquitination of purified GST-Vif protein in vitro (Fig. 4A). The ubiquitination of Vif by MDM2 was specific, as the omission of ubiqutin, E1, E2, or MDM2 prevented Vif-ubiquitination as shown in our previous experiments [13]. We also performed in vitro ubiquitination assays using immunopurified MDM2 and Cul5. Immunopurified MDM2 was able to induce ubiquitination of Vif in vitro to the same extent as Cul5 (Additional file 2, part A), while it could not ubiquitinate the N-terminal Vif deletion mutant $\Delta 22$ that was defective for binding MDM2 (Additional file 2, part B). These findings suggest that the interaction with MDM2 is important for Vif ubiquitination. We performed in vivo ubiquitination assays to further investigate the importance of MDM2 in Vif ubiquitination. Lysates of cells co-expressing Vif, either with an
MDM2 wild type (Wt) or a $\Delta \mathrm{RF}$ mutant, and His-tagged Ubiquitin (His-Ub) were analyzed for the presence of ubiquitinated Vif conjugates (Fig. 4B). Unfortunately, we detected a Vif band that non-specifically bound to Ni-NTA agarose (arrowhead) due to its nature as a sticky protein. Overexpression of MDM2 induced a ladder detected by anti-Vif $\mathrm{Ab}$, even in the absence of His-Ub (lane 2), suggesting that this ladder represented Vif protein polyubiquitinated with endogenous Ub (arrows with asterisk). Furthermore, in the presence of His-Ub, we detected a doublet of ladder which presumably represented Vif protein polyubiquitinated with endogenous and His-tagged Ub (arrows with asterisk and arrows, respectively). We also obtained similar results using a UbiQapture ${ }^{\mathrm{TM}}-\mathrm{Q}$ Kit (data not shown). We thus concluded that the overexpres- 

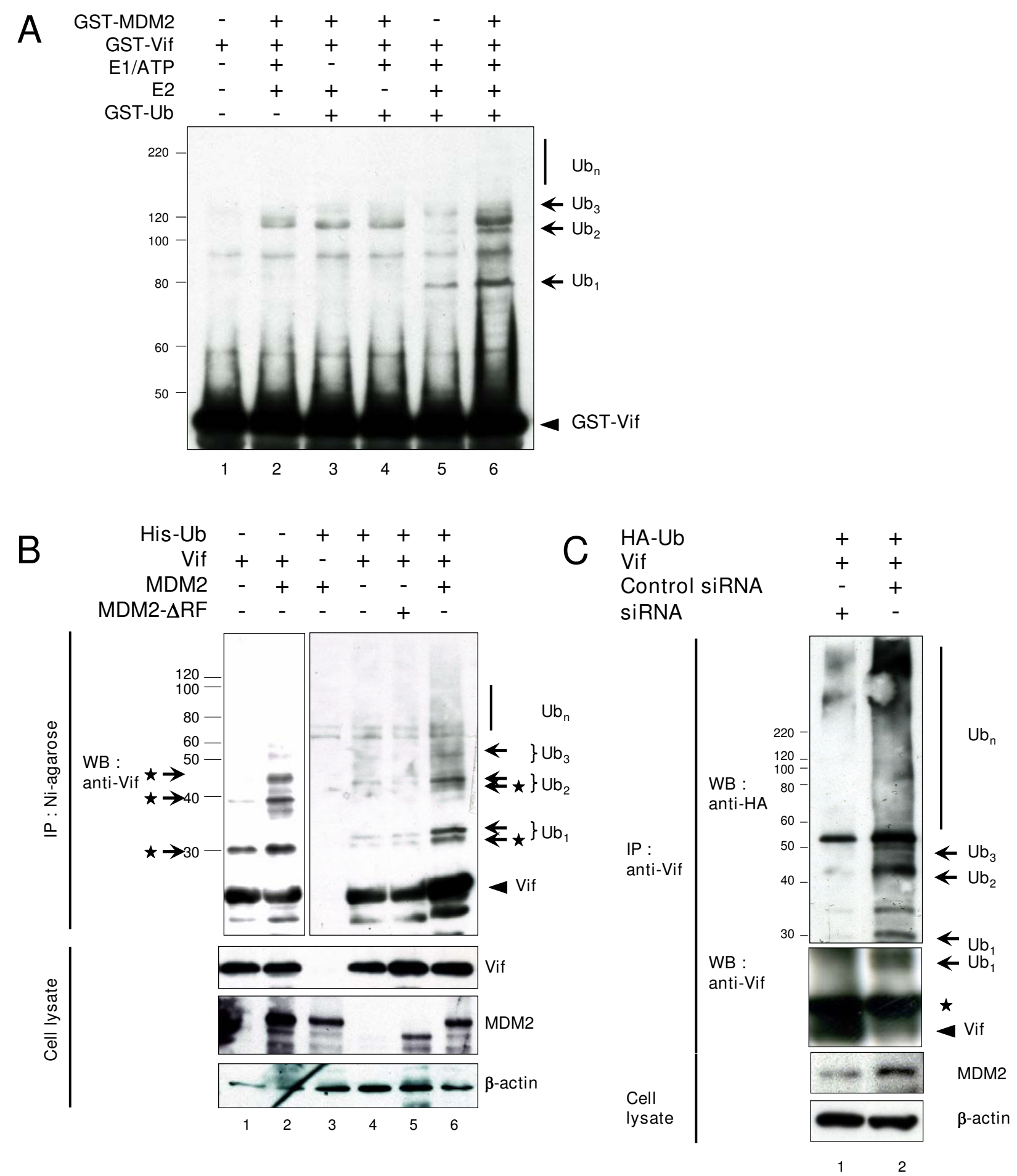

Figure 4 (see legend on next page) 
Figure 4 (see previous page)

MDM2 induced the polyubiquitination of Vif in vitro and in vivo. (A) GST-MDM2 induced the polyubiquitination of Vif in vitro. Bacterially expressed GST-Vif was subjected to in vitro ubiquitination assays. The reaction was performed in the presence or absence of EI, E2, GST-MDM2, and GST-Ubiquitin as indicated. Reactions were subjected to immunoblotting with anti-Vif $\mathrm{mAb}$. Arrows indicate GST-ubiquitin-conjugated Vif. (B) Overexpressed MDM2 induced the polyubiquitination of Vif in vivo. HEK293T cells were cotransfected with expression vectors for MDM2 Wt and a $\triangle$ RF mutant together with expression vectors for Vif and His-Ubiquitin (His-Ub) as indicated. Cells were treated with MGI32 for 6 hrs, and cell lysates were precipitated with Ni-NTA agarose beads followed by immunoblotting with the indicated Abs. Since Vif naturally bound to Ni-NTA agarose, we detected a Vif band itself (arrowhead), whereas no signal was detected in cells lacking Vif (lane 3). Arrows indicate His-Ub-conjugated Vif. Arrows with asterisk indicate Vif conjugated with endogenous ubiquitin. (C) Transduction of siRNA reduced cellular levels of endogenous MDM2 and polyubiquitination of Vif. HEK293T cells were cotransfected with expression vectors for MDM2 siRNA and control siRNA together with expression vectors for Vif and HA-Ubiquitin (HA-Ub). Cell lysates were immunoprecipitated with anti-Vif $\mathrm{mAb}$ followed by immunoblotting with the indicated Abs. Asterisk indicates immunoglobulin light chains from the immunoprecipitation.

sion of exogenous MDM2 efficiently induced polyubiquitination of Vif in vivo. Furthermore, the knock-down of endogenous MDM2 expression by introduction of MDM2-specific short interfering RNA (siRNA) resulted in a significant reduction in the amount of polyubiquitinated Vif, commensurate with the extent of reduced MDM2 expression (Fig. 4C). Collectively, these data indicated that MDM2 mediates polyubiquitination of Vif both in vitro and in vivo.

\section{MDM2 negatively regulates HIV-I replication in non- permissive cells through ubiqutination and degradation of Vif}

Next, we examined the effect of MDM2 on HIV-1 replication. In a single round infection assay (Fig. 5A), in the absence of $A 3 G$, viral replication was not affected by expression of MDM2 and/or Vif (lanes 1-6). In contrast, in the presence of $\mathrm{A} 3 \mathrm{G}$ in a non-permissive cell setting, without the expression of MDM2, the wild type virus could replicate but the $\Delta$ Vif virus could not, as previously reported (lanes $7 \& 8$ ) [3,8]. Co-expression of MDM2 reduced the cellular level of Vif (Fig. 5B, upper panel, lanes 5 \& 11), resulting in the increased virion incorporation of A3G (Fig. 5B, 2nd lower panel, lane 11 as compared with lanes 7 ) and the greater suppression of viral replication (Fig. 5A, lane 11 as compared with lane 7).

We also tested the effect of MDM2 on HIV-1 replication in the presence of A3F. MDM2 suppressed viral replication in the presence of $\mathrm{A} 3 \mathrm{~F}$, similar to results shown for $\mathrm{A} 3 \mathrm{G}$ (Additional file 3). These data indicated that the MDM2mediated Vif downregulation led to upregulated cellular A3G and A3F levels in producer cells, resulting in less infectious HIV-1 virions produced. Since MDM2 was previously reported to upregulate HIV-1 transcription by ubiquitination of Tat, we further examined HIV-1 replication in macrophages knocked down for MDM2 (Fig. 5C). We chose terminally differentiated macrophages as the target, because the knockdown of MDM2 is lethal for pro- liferating cells. HIV-1 replicated more efficiently in macrophages transfected with MDM2 siRNA than in control siRNA-transfected macrophages. These data indicated that MDM2 negatively regulated HIV-1 replication in non-permissive target cells through the ubiquitination and degradation of Vif.

To obtain further insights into the mechanisms why our MDM2 system did not induce the ubiquitination of A3G which was bound to Vif, we tested the expression levels and the binding affinity of A3G to Vif in transfected cells. Co-expression of MDM2 reduced the cellular levels of Vif and inversely increased the A3G levels in a dose dependent manner (Fig. 5D). Immunoprecipitation assays revealed that the co-expression of MDM2 blocked the binding of A3G to Vif in a dose dependent manner (Fig. $5 \mathrm{E})$. These data suggest that the interaction between MDM2 and Vif precludes A3G from binding to Vif.

\section{Discussion}

In this study, we report that MDM2 is a novel E3 ligase for HIV-1 Vif. MDM2 physically interacts with Vif and functions as an E3 ligase for Vif to induce its polyubiquitination and proteasomal degradation. Several E3 ligases including Cul5 [17], Nedd4, and AIP4 [18], have been reported to induce Vif ubiquitination, and the roles of Cul5 for Vif ubiquitination and degradation are especially well documented. Dang et al. have recently reported that Cul5 induces A3G degradation not by direct ubiquination of A3G but indirectly through Vif ubiqutination and that polyubiquitinated Vif might serve as a vehicle to transport A3G into proteasomes for degradation [23]. In this manuscript, we show that MDM2 only targets Vif for degradation but not A3G, although MDM2 and Cul5 both induce Vif ubiquitination (Additional file 2, part A). MDM2 reduced cellular Vif levels and inversely increased A3G levels (Fig. 5B \&5D), unlike Cul5. One possible explanation is that the binding of MDM2 to Vif precluded A3G from binding Vif (Fig. 5E), whereas a Cul5-Vif complex 
A

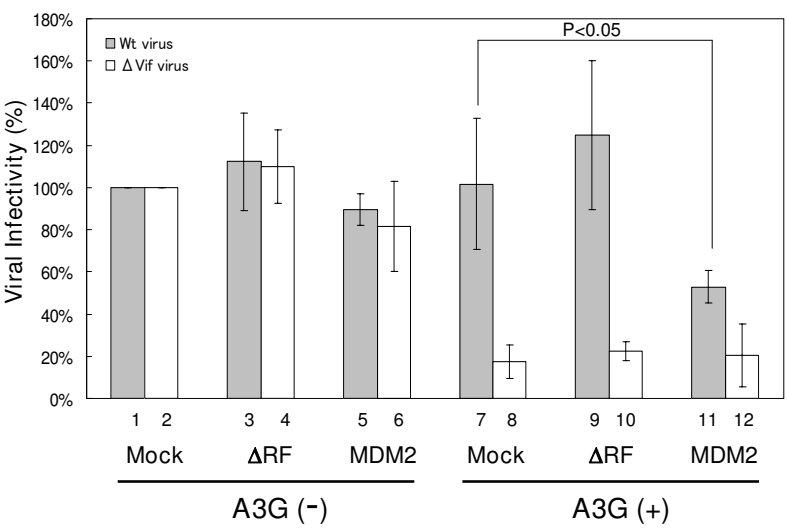

C

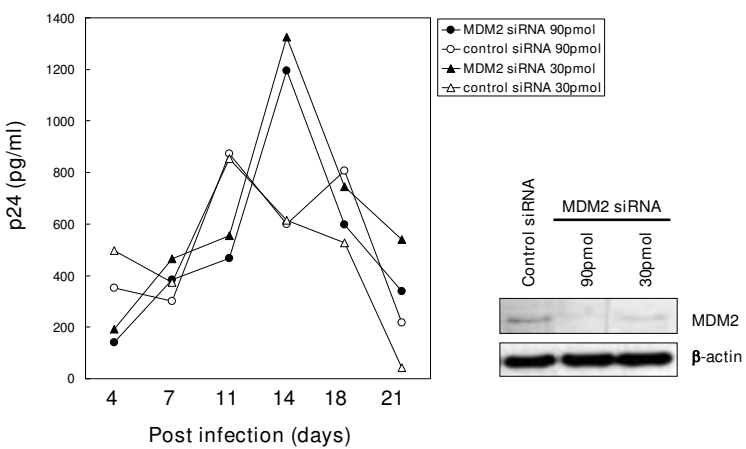

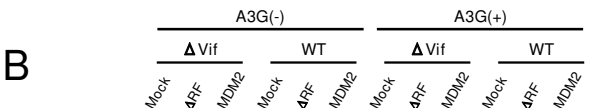
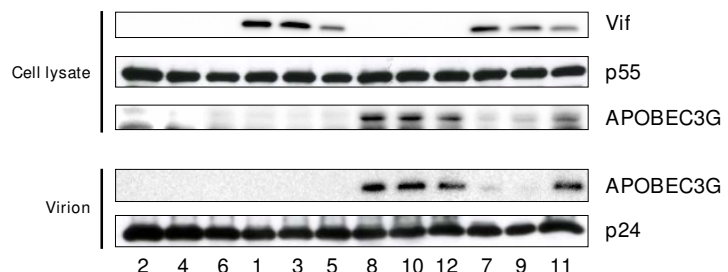

D

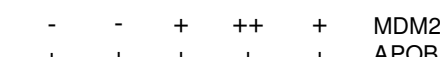

$++++\quad+$ APOBEC3G

$\begin{array}{llllll}+ & + & + & + & + & \text { APOB } \\ - & + & + & + & - & \text { Vif }\end{array}$
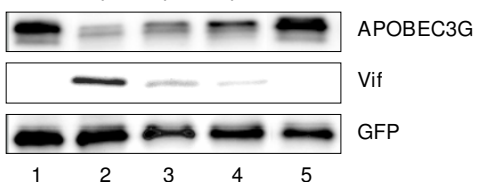

$E$

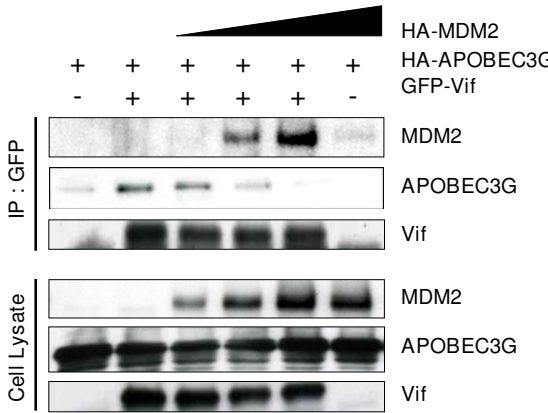

Figure 5

MDM2 negatively regulated HIV-I replication in non-permissive cells through the degradation of Vif. (A) The overexpression of MDM2 inhibited HIV-I replication in the presence of A3G. NL-43 Wt and $\Delta$ Vif viruses were produced from HEK293T cells transfected with expression vectors for MDM2 Wt and a $\triangle R F$ mutant in the presence or absence of $A 3 G$. The viral infectivity was examined using M8I66 cells. Values are presented as averages of more than 3 independent experiments. (B) MDM2 reduced cellular levels of Vif, resulting in more incorporation of A3G into HIV-I virions. Immunoblotting for cell lysates (upper 3 panels) and precipitated virions (lower 2 panels) was performed with the indicated Abs. Lane numbers correspond to those in Fig. 4A. (C) HIV-I replication in macrophages transfected with MDM2- and control-siRNA. MDM were transfected with MDM2- and control-siRNA and challenged with R5 HIV-I IR-FL (left panel). Cell lysates were subjected to immunoblotting with the indicated antibodies (right panels). (D) Coexpression of MDM2 reduced cellular levels of Vif and inversely increased A3G levels in a dose dependent manner. HEK293T cells were cotransfected with expression vectors for A3G, Vif, GFP, and MDM2 as indicated. Cell lysates were subjected to immunoblotting with the indicated Abs. (E) Immunoprecipitation assays revealed that the coexpression of MDM2 blocked the binding of A3G to Vif in a dose dependent manner. HEK293T cells were cotransfected with expression vectors for A3G, GFP-Vif, and MDM2 as indicated. Cell lysates were immunoprecipitated with anti-GFP mAb followed by immunoblotting with the indicated Abs.

can bind A3G to form a ternary complex. MDM2 binds the N-terminal region of Vif which does not overlap with, but is close to the A3G/A3F binding domain [25]. This binding might affect the interaction of Vif with A3G and/ or A3F. Furthermore, the evidence that an MDM2 $\triangle \mathrm{RF}$ mutant failed to protect A3G indicated that the ubiquitination and degradation of Vif is necessary to protect A3G and A3F from Vif. These findings suggest that different E3 ligases might play different roles in Vif ubiquitination. Further studies on the different roles of Vif ubiquitination by different E3 ligases and their virological significance should be investigated.

We demonstrate that MDM2 negatively regulated HIV-1 replication through Vif degradation. Through the degradation of target proteins (p53, pRB, etc), MDM2 can exert profound physiological effects on the regulation of cell cycle, cell proliferation, DNA repairs and other processes. To our knowledge, this is the first report to show that MDM2 plays an important role in viral replication 
through the degradation of viral proteins. Recently, MDM2 was also reported to ubiquitinate HIV-1 Tat protein and activate its transcriptional activity in a non-proteolytic manner [26]. Our experiment using MDM2 knockdown macrophages showed that HIV-1 replication in these macrophages was more efficient than in control siRNA-transfected macrophages. These data are consistent with MDM2 negatively regulateing HIV-1 replication through Vif ubiquitination (Fig. 5C). However, the replication efficiency of HIV-1 in MDM2 knockdown macrophages was only 2 -fold higher and was slower than in control siRNA-transfected macrophages. This suggests the possibilities that the ubiquitination of Tat might work as a positive regulatory factor at an earlier phase of infection and that MDM2 might be involved in both positive and negative regulation of HIV-1 replication at different stages. Further studies on the detailed effect of MDM2 on HIV-1 replication are needed.

We also demonstrated that Vif can bind MDM2 directly. We also mapped the interaction domain of MDM2 with Vif to amino acids 168-320 which is located in its central acidic and $\mathrm{Zn}$ finger domains. This central domain is different from the primary p53-binding site of MDM2 which is located in its N-terminal region; however, this central deomain was recently reported as a second p53-binding site and was shown to be important for the regulation of p53 stability [27-30] (Fig. 2B \&2C). Interestingly, several proteins including $\mathrm{p} 300, \mathrm{p} 14^{\mathrm{ARF}}$, and $\mathrm{pRB}$ bind to the central domain of MDM2 and regulate the stability and function of $\mathrm{p} 53$ via MDM2 [28,31]. Thus, it is possible that Vif might affect the stability and function of p53. Indeed, we confirmed that Vif can stabilize p53 (Izumi et al., unpublished data), which could explain why the effect of MDM2 on p53 degradation was weaker than that on Vif as shown in Fig. 1A. A further study is under way to elucidate this new function of Vif (Izumi et al., HIV-1 Vif induces G2 cell cycle arrest via the p53 pathway, unpublished).

Finally, expanding evidence suggests that the ubiquitination system plays important roles in many aspects of HIV1 replication including the degradation of A3G by Vif [911], the degradation of CD4 by Vpu [32], HIV-1 viral budding [33], Tat-mediated transactivation [26], and Vprinduced G2 cell cycle arrest [34,35]. The functional linkage between Vif and MDM2 also suggests that ubiquitin processes such as the A3G/Vif interplay is highly complex. It is obvious that HIV-1 replication in target CD4+ T cells is strongly affected by the interplay of these proteins. From the viral point of view, this interplay might give an advantage to HIV-1 replication. One possibility is that MDM2 regulates cellular Vif levels appropriately, such as not to affect viral replication [36] but just enough to antagonize A3G. Recent studies suggest that the G-to-A mutations induced by A3G may not be the mechanism by which A3G restricts or controls viral replication [37] and that a partially effective Vif inhibitor may actually accelerate the evolution of drug resistance and immune escape [38]. The inhibitory activity of MDM2 toward Vif could be partially effective and therefore could lead to viral evolution of drug resistance and immune escape. More recently, Nathans et al. have reported a small molecule that specifically antagonizes Vif function and inhibits viral replication by targeting the A3G/Vif axis. This compound enhances Vif degradation only in the presence of $A 3 G$, but does not induce A3G degradation and rather stabilizes A3G. They suggested the possibility of a new proteolytic enzyme for Vif degradation and that their new compound interferes with Vif interaction with a host protein in a VifA3G-host protein complex, thereby making Vif less stable. The precise biological significance of this Vif-A3G-host protein complex requires future elucidation. Nevertheless, modification or intervention of such Vif-A3G-host protein interplay could lead to the development of new therapeutic strategies for HIV-1 infection.

\section{Conclusion}

MDM2 is a novel E3 ligase for Vif which induces the polyubiquitination and degradation of Vif to negatively regulate HIV-1 replication.

\section{Methods \\ Plasmid constructs}

Expression vectors for hemagglutinin (HA)- or FLAGtagged MDM2, pCMV4/HA-MDM2 or pCMV4/FLAGMDM2, and their mutants were constructed as previously described [19]. An expression vector for HA-tagged human APOBEC3G, pcDNA3/HA-hA3G [39], and HIV-1 reporter plasmids, pNL43/Aenv-Luc (WT) and pNL43/ $\Delta$ env $\Delta$ vif-Luc ( $\Delta$ Vif) [8], were constructed as previously described. Expression vectors for FLAG-tagged Parkin and Cul5 (pcDNA3/FLAG-Parkin and pcDNA3/FLAG-Cul5, respectively) were constructed by the PCR method. Complementary DNA for HIV-1 Vif was also cloned into pDON-AI (TAKARA BIO INC.) and pDON/EGFP for expression of Vif and EGFP-fused Vif (EGFP-Vif). The subgenomic expression vector pNL-A1, which expresses all HIV-1 proteins except for gag and pol products, and its mutants expressing Vif deletion mutants were kind gifts from Dr. K. Strebel [22].

\section{Co-immunoprecipitation assays}

We performed an immunoprecipitation assay for proteinprotein interaction in vivo, as described previously [8]. HEK293T cells were cotransfected with pCMV4/HAMDM2 and pNL-A1 by the calcium phosphate method. Two days after transfection, cells were lysed in lysis buffer (25 mM HEPES pH7.4/150 mM NaCl/1 mM MgCl$/ 2 / 0.5 \%$ TritonX-100/10\% Glycerol) and complexes were immunoprecipitated with anti-MDM2 monoclonal antibody 
(mAb) (SMP-14, Santa Cruz Biotechnology, Inc., Santa Cruz, CA and Ab-1, Calbiochem, EMD Biosciences, Inc, Darmstadt, Germany) and Protein A-Sepharose beads (Amersham Biosciences Corp.) at $4^{\circ} \mathrm{C}$. The beads were washed with RIPA buffer (50 mM Tris-HCl pH8.0/150 $\mathrm{mM} \mathrm{NaCl} / 1 \%$ Triton-X 100/0.1\% SDS/0.1\% DOC) and analyzed by immunoblotting with anti-Vif mAb (\#319) (A kind gift from Dr. M. Malim through the AIDS Research and Reference Reagent Program) [40] or anti-HA $\mathrm{mAb}$ (12CA5). To map the regions of MDM2 necessary for binding to Vif, HEK293T cells were cotransfected with expression vectors for a series of MDM2 deletion mutants together with pNL-A1. Complexes were immunoprecipitated with anti-HA mAb and analyzed by immunoblotting with anti-Vif $\mathrm{mAb}$. To map the regions of Vif necessary for binding to MDM2, HEK293T cells were cotransfected with expression vectors for a series of Vif deletion mutants together with pCMV4/HA-MDM2. Complexes were immunoprecipitated with anti-Vif mAb and analyzed by immunoblotting with anti-MDM2 mAb. In all these experiments, transfected cells were treated with MG132 for 6 hrs prior to harvesting in order to stabilize both Vif and MDM2; otherwise we could not detect the expression of MDM2 because of its rapid degradation, as seen in Fig. 1A.

\section{In vitro and in vivo ubiquitination assays}

In vitro ubiquitination assays were carried out in ubiquitin reaction buffer $(50 \mathrm{mM}$ Tris- $\mathrm{HCl} / 2 \mathrm{mM}$ ATP/5 mM $\mathrm{MgCl}_{2} / 2 \mu \mathrm{M}$ DTT) with E1(200 ng), E2(Ubc5c)(150 ng), and GST-tagged ubiquitin (GST-Ub) $(10 \mu \mathrm{g})$ as described previously [13]. MDM2 and Vif were expressed as GSTfusion proteins in Escherichia coli strain DH5 $\alpha$ and BL21, respectively. The reactions were incubated at $30^{\circ} \mathrm{C}$ for 90 min. The samples were subjected to immunoblotting with anti-Vif mAb to detect GST-ubiquitin conjugated Vif.

For in vivo ubiquitination assays, HEK 293T cells were cotransfected with plasmids expressing Vif, FLAG-MDM2 or its mutants, and His-tagged ubiquitin (His-Ub) as indicated. Cells were treated with $10 \mu \mathrm{M}$ MG132 for 6 hrs prior to harvesting. Forty-eight hours post transfection, cell lysates were affinity-purified with Ni-NTA-agarose beads (Invitrogen corporation, Carlsbad, CA) and analyzed by immunoblotting with anti-Vif mAb.

For production of RNAi within the cells, we used the pSuper vector as described previously [19]. pSuper-MDM2-1 contained the $19 \mathrm{nt}$ derived from the $m d m 2$ cDNA (nt 404-422) as the target sequence. Double-stranded RNA containing scrambled $19 \mathrm{nt}$ was used as a control. HEK293T cells were transfected with pSuper plasmids together with plasmids expressing Vif and HA-Ub. Cell lysates were immunoprecipitated with anti-Vif $\mathrm{mAb}$ followed by immunoblottimg with anti-HA mAb.

\section{Single round infection assays with HIV-I luciferase reporter virus}

Luciferase reporter viruses with or without Vif were pre-

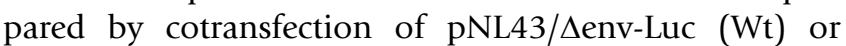
pNL43/ $\Delta$ env $\Delta$ vif-Luc ( $\Delta$ Vif) plus pVSV-G together with a mock vector or an expression vector for MDM2 or a mutant in the presence or absence of pcDNA3/hA3G by calcium phosphate as previously described [8]. The reporter viruses were adjusted according to p24 values and used to infect M8166 target cells. Productive infection was measured by luciferase activity and values were presented as percent infectivity relative to the value of each virus without the expression of hA3G.

\section{Knockdown of MDM2 in macrophages and replication assays}

Monocyte-derived macrophages (MDM) were cultured for 7 days from CD14+ monocytes isolated from the peripheral blood of an HIV-1-negative healthy individual. Electroporation with Stealth Select RNAi for MDM2 or Control (Invitrogen Corporation) was performed using the Nucleofector machine (Amaxa Inc., Gaithersburg, MD) according to the manufacturer's instructions. Twenty four hours after transfection, MDM were challenged with R5 HIV-1 $1_{\text {IR-FL }}$ at multiplicity of infection of 0.1 at $37^{\circ} \mathrm{C}$ for 3 hrs. The cells were cultured from day 4 to 21 after infection, and the concentration of p24 antigen in the supernatant was measured with an HIV-1 p24 antigen enzymelinked immunosorbent assay [ELISA] kit (ZeptMetrix, Buffalo, NY).

\section{Competing interests}

The authors declare that they have no competing interests.

\section{Authors' contributions}

TI. designed research, performed research, contributed vital new reagents, analyzed data, and wrote the paper. ATK designed research, analyzed data, wrote the paper, and organized the research. KS, KIo, and MM prepared the materials and performed a part of the research. KIwai, HK, TS, MT, SI., and HA contributed vital new reagents. YK contributed vital new reagents, performed a part of the research, and analyzed the data. $\mathrm{HH}$, KItoh, and JF designed the research, contributed vital new reagents, and analyzed the data. TU analyzed the data, drafted the paper, and organized the research. 


\section{Additional material}

\section{Additional file 1}

Supplementary figure 1 - the stability of Vif protein in p53-/- MEF and p53-/-MDM2-/- MEF cells. MEF cells were transfected with $p D O N /$ Vif or pcDNA3/HA-A3G. Twenty-two hours after transfection, the cells were treated with cycloheximide (CHX) for the indicated times, and cell lysates were subjected to immunoblotting with the indicated Abs.

Click here for file

[http://www.biomedcentral.com/content/supplementary/17424690-6-1-S1.pdf]

\section{Additional file 2}

Supplementary figure 2 - immunopurified MDM2 induced the polyubiquitination of Vif in vitro. (A) MDM2 as well as Cul5 induced the polyubiquitination of Vif. HEK293T cells were transfected with expression vectors for His-MDM2 and His-Cul5. His-tagged proteins were purified using Ni-NTA agarose and subjected to in vitro ubiquitination assays as described in a legend to Fig. 4A. Reactions were subjected to immunoblotting with anti-Vif Ab. Arrows indicate GST-Ub-conjugated Vif. Asterisks indicate non-specific bands associated with GST-Vif protein recognized by anti-Vif $A b$, as they are seen in lanes 1 and 3. (B) MDM2 induced the polyubiquitination of Vif Wt but not that of 422 that was defective for binding MDM2. Filled asterisks indicate non-specific bands associated with GST-Vif protein, while white asterisks indicate those associated with GST-Vif $\triangle 22$.

Click here for file

[http://www.biomedcentral.com/content/supplementary/17424690-6-1-S2.pdf]

\section{Additional file 3}

Supplementary figure 3 - the overexpression of MDM2 inhibited HIV1 replication in the presence of A3F. Single round infection assays were performed in the presence or absence of $A 3 F$ as described in a legend to Fig. 5A. Values are presented as averages of more than 3 independent experiments.

Click here for file

[http://www.biomedcentral.com/content/supplementary/17424690-6-1-S3.pdf]

\section{Acknowledgements}

We thank Drs. K. Strebel for the pNL-AI plasmid and its derivative mutants, D. P. Lane for $\mathrm{p} 53^{-/-M D M 2-/-D K O-M E F, ~ a n d ~ M . ~ M a l i m ~ f o r ~ t h e ~ a n t i-~}$ Vif mAb (\#319) through the AIDS Research and Reference Reagent Program, Division of AIDS, NIAID, NIH. This study was partly supported by grants-in-aid from the Ministry of Education, Culture, Sports, Science, and Technology, from the Ministry of Health, Labour and Welfare, Japan, from the Naito Foundation, and from Mitsubishi Pharma Research Foundation.

\section{References}

I. Goff SP: Retrovirus restriction factors. Mol Cell 2004, 16:849-859.

2. Towers G]: The control of viral infection by tripartite motif proteins and cyclophilin A. Retrovirology 2007, 4:40.

3. Sheehy AM, Gaddis NC, Choi JD, Malim MH: Isolation of a human gene that inhibits HIV-I infection and is suppressed by the viral Vif protein. Nature 2002, 418:646-650.

4. Goila-Gaur R, Strebel K: HIV-I Vif, APOBEC, and intrinsic immunity. Retrovirology 2008, 5:5I.

5. Mangeat B, Turelli P, Caron G, Friedli M, Perrin L, Trono D: Broad antiretroviral defence by human APOBEC3G through lethal editing of nascent reverse transcripts. Nature 2003, 424:99-103.

6. Harris RS, Bishop KN, Sheehy AM, Craig HM, Petersen-Mahrt SK, Watt IN, Neuberger MS, Malim MH: DNA deamination mediates innate immunity to retroviral infection. Cell 2003, I 1 3:803-809.

7. Zhang H, Yang B, Pomerantz RJ, Zhang C, Arunachalam SC, Gao L: The cytidine deaminase CEMI5 induces hypermutation in newly synthesized HIV-I DNA. Nature 2003, 424:94-98.

8. Shindo K, Takaori-Kondo A, Kobayashi M, Abudu A, Fukunaga K, Uchiyama T: The enzymatic activity of CEMI5/Apobec-3G is essential for the regulation of the infectivity of HIV-I virion but not a sole determinant of its antiviral activity. J Biol Chem 2003, 278:444I2-444I6.

9. Marin M, Rose KM, Kozak SL, Kabat D: HIV-I Vif protein binds the editing enzyme APOBEC3G and induces its degradation. Nat Med 2003, 9: 1398-I403.

10. Sheehy AM, Gaddis NC, Malim MH: The antiretroviral enzyme APOBEC3G is degraded by the proteasome in response to HIV-I Vif. Nat Med 2003, 9: I 1404-I 407.

II. Stopak K, de Noronha C, Yonemoto W, Greene WC: HIV-I Vif blocks the antiviral activity of APOBEC $3 \mathrm{G}$ by impairing both its translation and intracellular stability. Mol Cell 2003, I 2:59|-60|

12. Yu X, Yu Y, Liu B, Luo K, Kong W, Mao P, Yu XF: Induction of APOBEC3G ubiquitination and degradation by an HIV-I VifCul5-SCF complex. Science 2003, 302: I056-1060.

13. Kobayashi M, Takaori-Kondo A, Miyauchi Y, Iwai K, Uchiyama T: Ubiquitination of APOBEC3G by an HIV-I Vif-Cullin5Elongin B-Elongin C Complex Is Essential for Vif Function. J Biol Chem 2005, 280:18573-18578.

14. Zheng Y-H, Irwin D, Kurosu T, Tokunaga K, Sata T, Peterlin BM: Human APOBEC3F Is Another Host Factor That Blocks Human Immunodeficiency Virus Type I Replication. J Virol 2004, 78:6073-6076.

15. Shirakawa K, Takaori-Kondo A, Kobayashi M, Tomonaga M, Izumi T, Fukunaga K, Sasada A, Abudu A, Miyauchi Y, Akari H: Ubiquitination of APOBEC3 proteins by the Vif-Cullin5-ElonginBElonginC complex. Virology 2006, 344:263-266.

16. Fujita M, Akari H, Sakurai A, Yoshida A, Chiba T, Tanaka K, Strebel K, Adachi A: Expression of HIV-I accessory protein Vif is controlled uniquely to be low and optimal by proteasome degradation. Microbes Infect 2004, 6:791-798.

17. Mehle A, Goncalves J, Santa-Marta M, McPike M, Gabuzda D: Phosphorylation of a novel SOCS-box regulates assembly of the HIV-I Vif-Cul5 complex that promotes APOBEC3G degradation. Genes Dev 2004, 18:286I-2866.

18. Dussart S, Courcoul M, Bessou G, Douaisi M, Duverger Y, Vigne R, Decroly $E$ : The Vif protein of human immunodeficiency virus type $I$ is posttranslationally modified by ubiquitin. Biochem Biophys Res Commun 2004, 3 I 5:66-72.

19. Higashitsuji H, Itoh K, Sakurai T, Nagao T, Sumitomo Y, Masuda T, Dawson S, Shimada Y, Mayer RJ, Fujita J: The oncoprotein gankyrin binds to MDM2/HDM2, enhancing ubiquitylation and degradation of p53. Cancer Cell 2005, 8:75-87.

20. Honda $\mathrm{R}$, Tanaka $\mathrm{H}$, Yasuda $\mathrm{H}$ : Oncoprotein MDM2 is a ubiquitin ligase E3 for tumor suppressor p53. FEBS Lett 1997, 420:25-27.

21. Yu Y, Xiao Z, Ehrlich ES, Yu X, Yu X-F: Selective assembly of HIVI Vif-Cul5-ElonginB-ElonginC E3 ubiquitin ligase complex through a novel SOCS box and upstream cysteines. Genes Dev 2004, 1 8:2867-2872.

22. Strebel K, Daugherty D, Clouse K, Cohen D, Folks T, Martin MA: The HIV 'A' (sor) gene product is essential for virus infectivity. Nature 1987, 328:728-730.

23. Dang $Y$, Siew LM, Zheng YH: APOBEC3G is degraded by the proteasomal pathway in a Vif-dependent manner without being polyubiquitylated. I Biol Chem 2008, 283:|3|24-|3|3|.

24. Honda R, Yasuda H: Activity of MDM2, a ubiquitin ligase, toward p53 or itself is dependent on the RING finger domain of the ligase. Oncogene 2000, 19:1473-1476.

25. He Z, Zhang W, Chen G, Xu R, Yu XF: Characterization of conserved motifs in HIV-I Vif required for APOBEC3G and APOBEC3F interaction. I Mol Biol 2008, 38 I: 1000-I0II.

26. Brès $\mathrm{V}$, Kiernan RE, Linares LK, Chable-Bessia $C$, Plechakova $O$, Tréand C, Emiliani S, Peloponese JM, Jeang KT, Coux O, Scheffner M, Benkirane M: A non-proteolytic role for ubiquitin in Tat-medi- 
ated transactivation of the HIV-I promoter. Nat Cell Biol 2003, 5:754-76I.

27. Argentini $M$, Barboule N, Wasylyk $B$ : The contribution of the acidic domain of MDM2 to p53 and MDM2 stability. Oncogene 200I, 20:1267-1275.

28. Iwakuma T, Lozano G: MDM2, an introduction. Mol Cancer Res 2003, I:993-1000.

29. Kawai H, Wiederschain D, Yuan ZM: Critical contribution of the MDM2 acidic domain to p53 ubiquitination. Mol Cell Biol 2003, 23:4939-4947.

30. Meulmeester E, Frenk R, Stad R, de Graaf P, Marine JC, Vousden KH, Jochemsen AG: Critical role for a central part of Mdm2 in the ubiquitylation of p53. Mol Cell Biol 2003, 23:4929-4938.

31. Ganguli G, Wasylyk B: p53-independent functions of MDM2. Mol Cancer Res 2003, I: I 027-I035.

32. Margottin F, Bour SP, Durand H, Selig L, Benichou S, Richard V, Thomas $D$, Strebel K, Benarous R: A novel human WD protein, $h-$ beta $\operatorname{TrCp}$, that interacts with HIV-I Vpu connects CD4 to the ER degradation pathway through an F-box motif. Mol Cell 1998, I:565-574.

33. Freed EO: Viral late domains. J Virol 2002, 76:4679-4687.

34. Wen X, Duus KM, Friedrich TD, de Noronha CM: The HIVI protein $\mathrm{Vpr}$ acts to promote $\mathbf{G} 2$ cell cycle arrest by engaging a DDBI and Cullin4A-containing ubiquitin ligase complex using VprBP/DCAFI as an adaptor. I Biol Chem 2007, 282:27046-27057.

35. Schrofelbauer B, Hakata Y, Landau NR: HIV-I Vpr function is mediated by interaction with the damage-specific DNAbinding protein DDBI. Proc Natl Acad Sci USA 2007, 104:4|30-4|35.

36. Akari H, Fujita $M$, Kao $S$, Khan MA, Shehu-Xhilaga M, Adachi $A$, Strebel K: High level expression of human immunodeficiency virus type-I Vif inhibits viral infectivity by modulating proteolytic processing of the Gag precursor at the p2/nucleocapsid processing site. J Biol Chem 2004, 279: I 2355- I 2362.

37. Ulenga NK, Sarr AD, Hamel D, Sankale JL, Mboup S, Kanki PJ: The level of APOBEC3G (hA3G)-related G-to-A mutations does not correlate with viral load in HIV type I-infected individuals. AIDS Res Hum Retroviruses 2008, 24: I 285-I290.

38. Pillai SK, Wong JK, Barbour JD: Turning up the volume on mutational pressure: is more of a good thing always better? (A case study of HIV-I Vif and APOBEC3). Retrovirology 2008, 5:26.

39. Kobayashi M, Takaori-Kondo A, Shindo K, Abudu A, Fukunaga K, Uchiyama T: APOBEC3G Targets Specific Virus Species. J Virol 2004, 78:8238-8244.

40. Simon JH, Southerling TE, Peterson JC, Meyer BE, Malim MH: Complementation of vif-defective human immunodeficiency virus type I by primate, but not nonprimate, lentivirus vif genes. J Virol 1995, 69:4166-4172.

Publish with Bio Med Central and every scientist can read your work free of charge

"BioMed Central will be the most significant development for disseminating the results of biomedical research in our lifetime. "

Sir Paul Nurse, Cancer Research UK

Your research papers will be:

- available free of charge to the entire biomedical community

- peer reviewed and published immediately upon acceptance

- cited in PubMed and archived on PubMed Central

- yours - you keep the copyright
BioMedcentral 